\title{
A Study of Equilibrium in Argon Arcs
}

\author{
J. B. Shumaker and C. H. Popenoe
}

\author{
Institute for Basic Standards, National Bureau of Standards, Washington, D.C. 20234
}

(November 8, 1971)

\begin{abstract}
The intensities of the $7147 \AA$ Ar I and $4806 \AA$ Ar II lines obtained from wall-stabilized arcs at pressures of 0.2 to $5 \mathrm{~atm}$ are presented in an Olsen-Richter diagram. Departures from equilibrium are evident at electron densities below $5 \times 10^{16} \mathrm{~cm}^{-3}$. The assumption of equilibrium at higher electron densities leads to transition probabilities in fair agreement with lifetime measurements.
\end{abstract}

Key words: Argon arcs; argon transition probabilities; equilibrium; LTE.

\section{Introduction}

For the measurement of optical transition probabilties and the Stark widths and shifts of spectral lines in plasmas no spectral source has been as fruitful as the high current atmospheric pressure stabilized arc. And of all the elements studied by this technique none has been more thoroughly worked over than argon. It is therefore a matter of considerable concern that different plasma diagnostic techniques lead to different transition probability values in argon arcs $[1,2,3] .{ }^{1}$ The two plasma diagnostic techniques which have been used to establish absolute scales for argon transition probabilities in arcs are various modifications of the FowlerMilne method [4-7] and the hydrogen line shape method $[2,8,9.10]$. The latter method leads to systematically larger transition probabilities: by about 30 percent for Ar I lines and by nearly a factor of 2 for Ar II lines. Other independent determinations of these transition probabilities such as theoretical calculations and lifetime measurements generally tend to support the higher values for Ar I and the lower values for Ar II. Since these discrepancies may be symptomatic of nonequilibrium behavior of the ares it is important to discover whether local thermodynamic equilibrium (LTE) is a characteristic of these experiments because if not, then the large amounts of atomic data being generated by the arc technique are of doubtful value.

If we apply LTE validity criteria estimates $[11,12]$ to the usual experimental conditions existing in argon arcs, we find that for electron densities greater than $5 \times 10^{16} \mathrm{~cm}^{-3}$ or so we may expect all neutral and singly ionized populations to be within at most 10 percent of their LTE values. Unfortunately, this LTE threshold estimation is uncertain by perhaps half an order of

\footnotetext{
' Figures in brackets indicate the literature references at the end of this paper.
}

magnitude because of the approximate nature of the theoretical arguments and the rough estimates of collision cross sections. Experimental attempts to define the LTE threshold electron density have thus far not convincingly removed any of this uncertainty. The experimental values vary from $5 \times 10^{14}$ (Bourasseau, et al.) [13] to $10^{18} \mathrm{~cm}^{-3}$ (Bober and Tankin) [6] with most of the measurements suggesting values in the range from $2 \times 10^{15}$ to $10^{16} \mathrm{~cm}^{-3}$ [14-17]. A recent detailed theoretical treatment of the argon arc by Uhlenbusch, Fischer, and Hackmann [18] is in general accord with the Bober and Tankin value.

In this paper we present argon arc data which show distinct departures from LTE at electron densities below $5 \times 10^{16} \mathrm{~cm}^{-3}$ and we discuss briefly the evidence that LTE prevails at higher electron densities and the relationship of this problem to hydrogen line broadening theory and to argon lifetime measurements.

\section{Experimental Procedure}

Our data consist of spectral intensity measurements of two well-isolated, optically thin argon lines. The measurements were performed side-on in wallstabilized arcs. The arc chamber for most of the experiments was of the watercooled copper disk type developed by Maecker [19]. Two channel diameters were used: $4.8 \mathrm{~mm}$ and $3.2 \mathrm{~mm}$. One experiment was performed with a $4.8 \mathrm{~mm}$ internal diameter fused silica tube arc chamber similar in principle to that described by Knopp and Cambel [20] in which the arc is observed within a transparent constricting tube rather than in the open gap between two copper constrictors.

We show in figure 1 our measurements of the intensities of the spectral lines $7147 \AA$ Ar I and $4806 \AA$ Ar II. At each of the five indicated pressures from 0.2 to 
$4.98 \mathrm{~atm}$ experiments were conducted at a number of arc currents. Under each operating condition the intensities of both spectral lines were recorded at a number of wavelengths encompassing the line and at generally 40 to 50 equally spaced points traversing the arc diameter. Following an Abel inversion to obtain local radial volume emission values the line intensities were computed as usual by subtracting the continuum, integrating over the line, and correcting for the distant line wings. Details of the data recording and reduction have been described previously [8]. The final result consists of corresponding pairs of intensities for the two spectral lines at a number of radial points for each arc experiment. These intensity pairs are plotted logarithmically in figure 1.

In figure 2 we show curves representing the relationship between the populations of the upper states of these two lines which would exist in complete thermodynamic equilibrium at the five pressures of our experiments. As a matter of interest we have plotted also contours of constant electron density and constant temperature. These curves were computed in accordance with Griem's treatment [11] of ionization potential lowering, partition function cut-off and pressure correction. Since the ratio of a line intensity to the upper state number density is a constant proportional to the transition probability and since on a logarithmic scale such a constant represents a simple translation it should be possible to fit the curves of figure 2 to the data of figure 1 if the arc data were obtained under LTE conditions [5]. The curves in figure 1 show such a fitting applied to the data at the three highest pressures. The fitting was performed numerically by a generalized nonlinear least squares fitting technique described by Wolberg [21]. The atomic transition probabilities obtained this way are $\mathrm{A}(7147 \mathrm{Ar} \mathrm{I})=$ $5.57 \pm 0.02 \times 10^{5} \mathrm{~s}^{-1}$ and $\mathrm{A}(4806 \mathrm{Ar}$ II $)=7.86 \pm 0.10 \times$ $10^{7} \mathrm{~s}^{-1}$. The borders of figure 2 have been selected according to these transition probabilities so as to permit superposition of figures 1 and 2 and estimation of electron density and temperature in the experimental data.

Although the least squares fitting was based only on the three highest pressures it is clear that the 0.5 atm data also agree well with this choice of transition probabilities. The $0.2 \mathrm{~atm}$ data on the other hand are in serious disagreement with these transition probabilities and, in fact, the scatter of data clearly precludes any meaningful fitting at this pressure.

Our data include no corrections for self-absorption. Based upon experimentally measured Stark broadening parameters $[7,22]$ and the continuum intensities and $A$ values measured here we estimate that the maximum error introduced by self-absorption is less than 1 percent for the $7147 \AA$ Ar I line in our highest current, highest pressure experiment and about 1 percent for the $4806 \AA$ Ar II line in our highest temperature atmospheric pressure experiment.

By comparing an arc with a black body in which complete LTE exists one can recognize three essential differences which may cause departures from equilibrium. First, in the arc much of the radiation escapes so that LTE will be achieved only if radiative depopulation rates are negligible compared to rates of collisional population and depopulation. Second, in the arc steep temperature and number density gradients exist which may generate diffusional fluxes of such magnitude that collisional (and radiative) processes

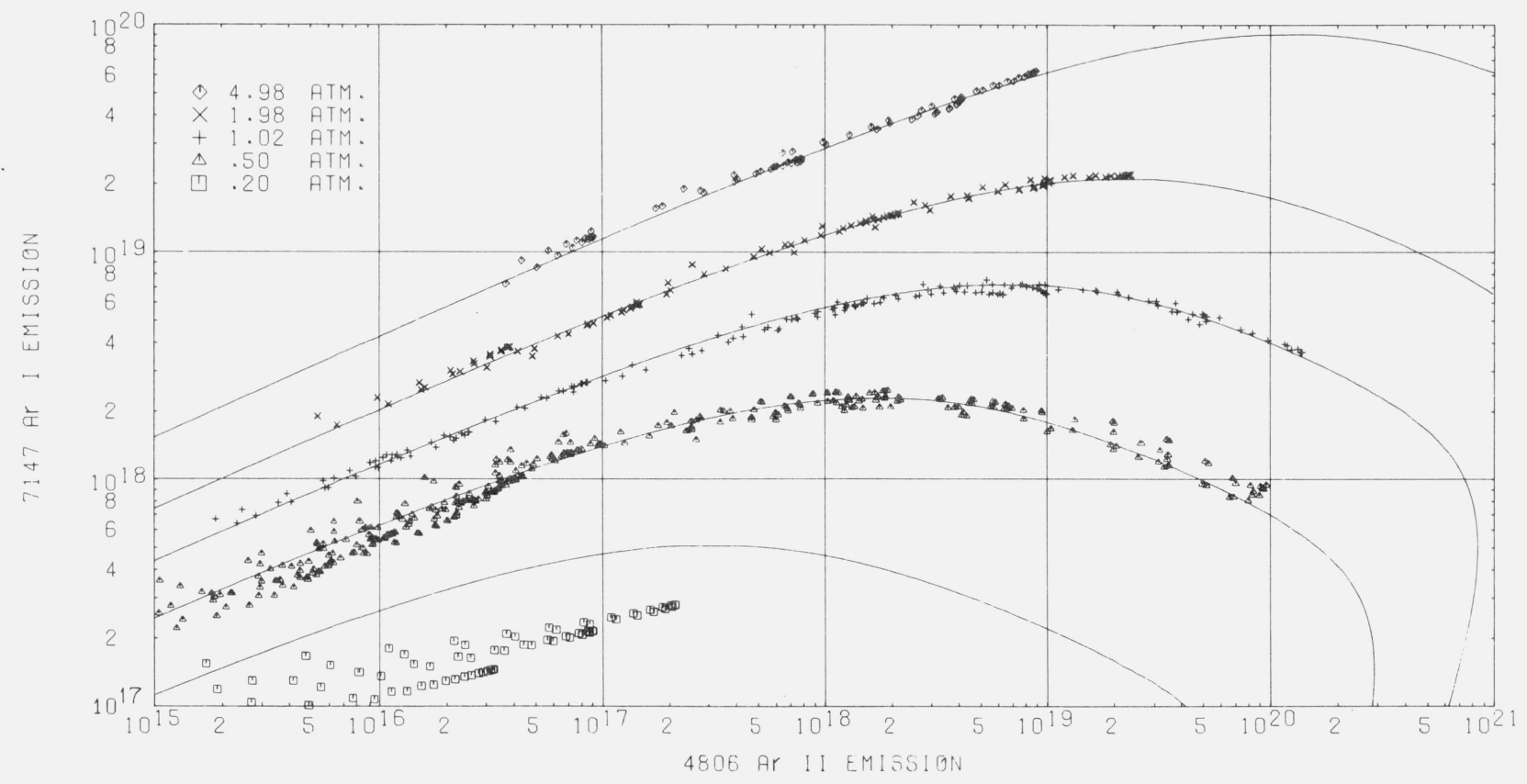

Figure 1. Argon arc line intensity measurements in photons $/\left(\mathrm{cm}^{3} \mathrm{~s}\right)$. 


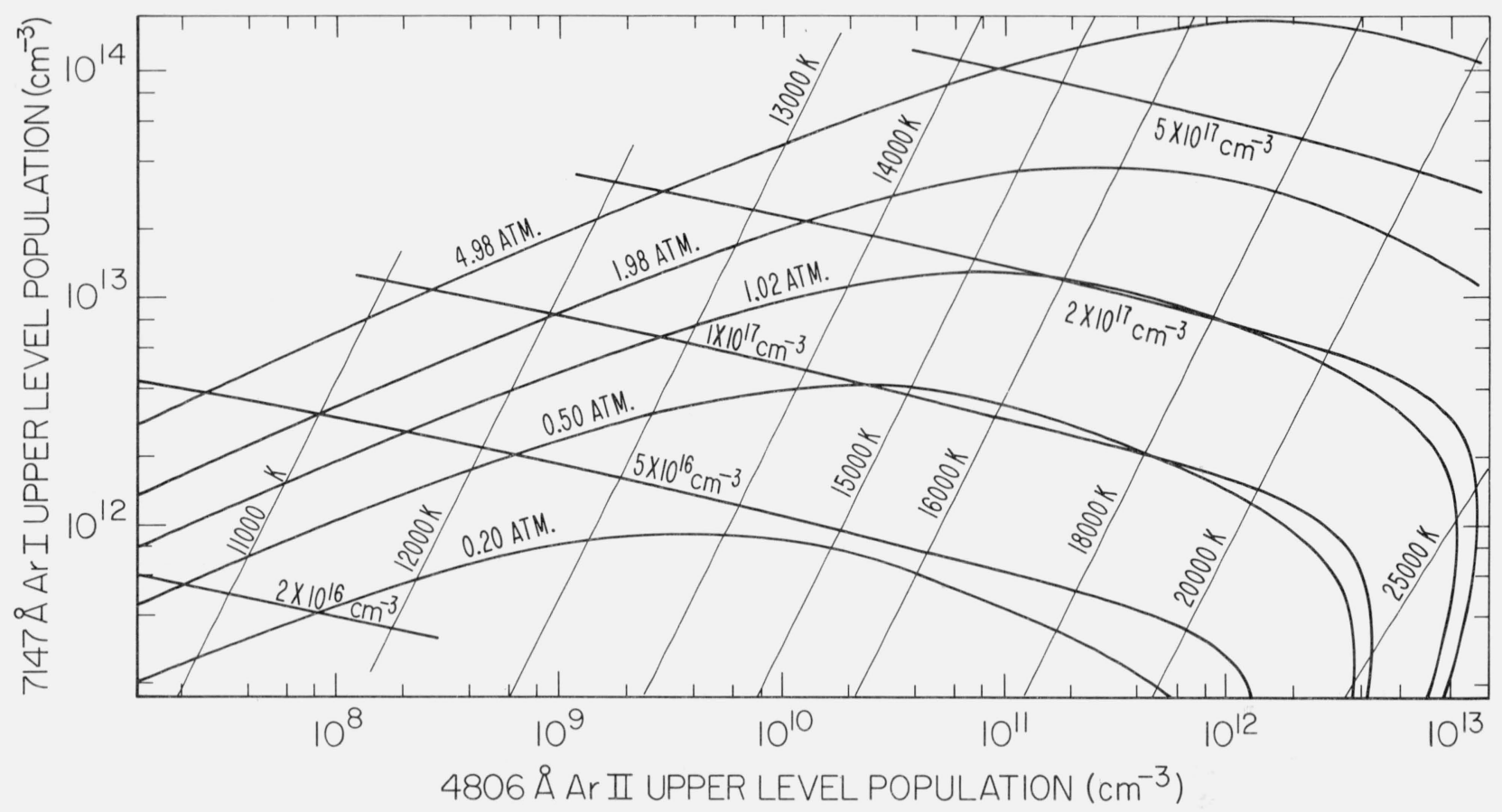

FIGURE 2. Computed equilibrium upper level populations for conditions of experiments of figure 1.

Also shown are constant electron density and constant temperature contours.

are unable to maintain equilibrium distributions. And third, since electrons absorb energy from an electric field much more rapidly than ions do the kinetic temperature of the electron gas will be higher than that of the heavy particle gas which obtains its energy then mainly by collisions with the electrons. Since collisional processes will be dominated by electron collisions it turns out that all of these effects should decrease rapidly in importance with increasing electron density $[11,12]$. The importance of the second effect should also depend upon arc channel diameter and radial position in the arc.

The data of figure 1 span a range in electron density from $10^{16}$ to $5 \times 10^{17} \mathrm{~cm}^{-3}$ and we see from the fitting of the LTE curves that there is a transition from a condition where no reasonable fitting is possible at low electron densities to a condition where an entirely satisfactory fit can be made at higher electron densities. This behavior is in accord with the qualitative electron density dependence arguments above and the assumption that the arc is in LTE at the higher electron densities.

At each pressure the arc currents for the successive experiments were chosen to furnish considerable overlap between the data obtained near the axis at one current and that obtained from the outer regions of the next higher current experiment. At the three higher pressures and over much of the range of the $0.5 \mathrm{~atm}$ data there is no significant systematic radially dependent discrepancy in these overlap regions. The data obtained from experiments with the two different channel diameters also overlap considerably. In the 1 atm data, for example the overlap in the $4806 \AA$ Ar II in- tensity is two orders of magnitude with the large channel providing data from $10^{15}$ to $10^{19} \mathrm{~cm}^{-3} \mathrm{~s}^{-1}$ and the small channel from $10^{17}$ to $10^{20} \mathrm{~cm}^{-3} \mathrm{~s}^{-1}$. The data show no evidence that the channel diameter influences the results at electron densities above $5 \times 10^{16} \mathrm{~cm}^{-3}$. At lower electron densities we have only the large channel data and cannot make the comparison. Our results, then are not inconsistent with the equilibrium hypothesis at the higher electron densities. The data in figure 1 do not include any measurements beyond a maximum radial distance which usually lay between 1.5 and $2 \mathrm{~mm}$. At that distance from the arc axis the $4806 \AA$ Ar II line intensity was generally about two orders of magnitude weaker than on axis and the remaining data were dominated by noise and scattered light.

The scatter of data in the lower left hand portion of figure 1 is caused by a dependence upon the radial point at which the measurement is made. This is shown in figure 3 where we have replotted the data from two typical experiments in this region. Data from the arc axis appear in the upper right of this figure and data from the outer regions appear at the lower left. It will be seen that the deviations from the LTE curves fitted at higher electron densities show a systematic radial behavior. At the arc center the data lie below or to the right of the curve whereas at large radii the data lie above or to the left of the curve. This behavior agrees qualitatively with that computed by Uhlenbusch [18] for a simple model in which the kinetic temperature of the heavy particles is allowed to be lower than that of the electrons, and the neutral atom ground state population is allowed to be larger 


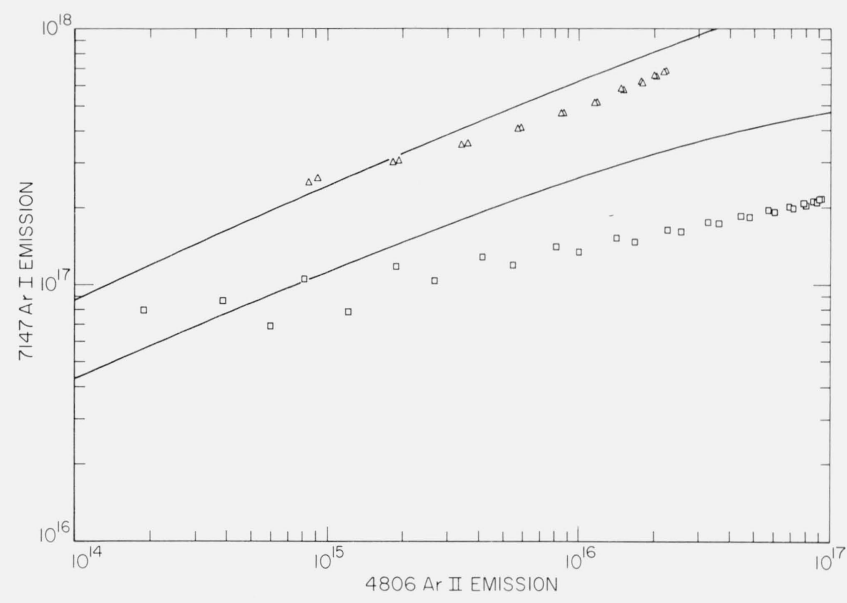

FigURE 3. Detail from figure 1 .

Two sub-atmospheric pressure argon arc experiments in $0.48 \mathrm{~cm}$ diameter channel: 0.5 atm, $47.2 \mathrm{amp}$ and $0.2 \mathrm{~atm}, 62.2 \mathrm{amp}$.

than the LTE value obtained from the electron temperature and from the free electron or excited state number densities.

Using an extension of the Uhlenbusch model in which we also include ionic ground state overpopulations, we can interpret these data in terms of small departures from LTE. For this purpose we modify the usual equilibrium equations for arc diagnostics which relate pressures and temperatures to the populations of the excited states and electrons as follows:

Boltzmann distributions

$$
\begin{array}{ll}
n_{0}^{j}=\frac{n_{0}^{0} g_{0}^{j}}{b_{0} g_{0}^{0}} \exp \left(-E_{0}^{j} / k T_{e}\right) & j>1 \\
n_{1}^{j}=\frac{n_{1}^{0} g_{1}^{j}}{b_{1} g_{1}^{0}} \exp \left(-E_{1}^{j} / k T_{e}\right) & j>1
\end{array}
$$

Saha equation

$$
\frac{n_{e} n_{1}^{0}}{n_{0}^{j}}=2\left(\frac{2 \pi m_{e} k T_{e}}{h^{2}}\right)^{3 / 2} \frac{g_{1}^{0}}{g_{0}^{j}} \exp \left(-\left(\chi_{0}-E_{0}^{j}\right) / k T_{e}\right) \quad j>1
$$

Electrical neutrality

$$
n_{e}=\sum_{j=0} n_{1}^{j}
$$

Dalton's Law

$$
\begin{aligned}
& P=\left(\sum_{j=0} n_{0}^{j}+\sum_{j=0} n_{1}^{j}\right) k T_{g}+n_{e} k T_{e}-\Delta P \\
& \quad=P^{\prime}-\left(n_{0}^{1}+n_{e}\right) k\left(T_{e}-T_{g}\right)+n_{0}^{0 \prime}\left(b_{0}-1\right) k T_{g}
\end{aligned}
$$

where

$$
\begin{aligned}
& n_{0}^{0^{\prime}}=n_{0}^{0} / b_{0} \\
& n_{0}^{\prime}=n_{0}^{0^{\prime}}+\sum_{j=1} n_{0}^{j} \\
& P^{\prime}=\left(n_{0}^{\prime}+2 n_{e}\right) k T_{e}-\Delta P
\end{aligned}
$$

In these equations the subscript 0 denotes a neutral atom; subscript 1 denotes the first ion; and higher ions have been neglected for simplicity. Superscript 0 denotes a ground state and other superscripts denote excited states. $n_{e}, m_{e}$, and $T_{e}$ are the electron number density, mass, and kinetic temperature. $\chi_{0}$ is the effective ionization energy, i.e., the ionization energy minus the lowering caused by the plasma interactions. $P$ is the pressure and $\Delta P$ is the small pressure correction for plasma interactions. $b_{0}$ and $b_{1}$ are measures of the degree of atomic and ionic ground state overpopulation. $T_{g}$ is the heavy particle kinetic temperature. We expect that $T_{e} \geqq T_{g}, b_{0} \geqq 1$, and $b_{1} \geqq 1$ with the equality sign characterizing the equilibrium condition.

In order to apply these equations to the data in figure 3 we introduce the approximation

$$
n_{e}=n_{1}^{0}
$$

and equations for the line intensities

$$
\begin{aligned}
& I_{0}^{j}=A_{0}^{j} n_{0}^{j} \text { photons }\left(\mathrm{cm}^{3} \mathrm{sec}\right)^{-1} \\
& I_{1}^{j}=A_{1}^{j} n_{1}^{j} \text { photons }\left(\mathrm{cm}^{3} \mathrm{sec}\right)^{-1}
\end{aligned}
$$

and for the intensity of the continuum which we measure near $4806 \AA$ :

$$
I_{\text {cont }}=1.032 \times 10^{-11} n_{e}^{2} \lambda^{-1} T_{e}^{-1 / 2} \xi \text { photons }\left(\mathrm{cm}^{3} \mathrm{~s}\right)^{-1}
$$

where $\lambda$ is the wavelength in angstroms and $\xi$ is a slowly varying function of $T_{e}$ and $\lambda$, whose value is of the order of magnitude of 1 . Now we can use equations 1 to 9 and the continuum and line intensity measurements to calculate the ionic ground state overpopulation factor $b_{1}$ directly. From our measurements we cannot separate the two contributions to $P-P^{\prime}$ (eq 5) but since the two effects operate in opposite directions we can obtain a lower bound to $b_{0}$ by assuming $T_{g}=T_{e}$. Actually the assumption $T_{q}=T_{e}$ probably doesn't introduce a serious error near the axis in these experiments. Kruger [23] has carried out a calculation similar to Uhlenbusch's for a 35 amp atmospheric pressure argon arc and finds $T_{e}-T_{g}=200 \mathrm{~K}$ at the arc axis where the electron density is about $10^{16}$. Uhlenbusch [18] finds a similar value for 200 amperes and an electron density of $1.5 \times 10^{17}$. The evaluation of $b_{1}$ and $b_{0}{ }^{\mathrm{min}}$ from these equations requires values for the transition probabilities and $\xi\left(T_{e}, 4806\right)$. If LTE is assumed in our high pressure data these values can be calculated from these data. For this case the $\mathrm{A}$ values have been given 


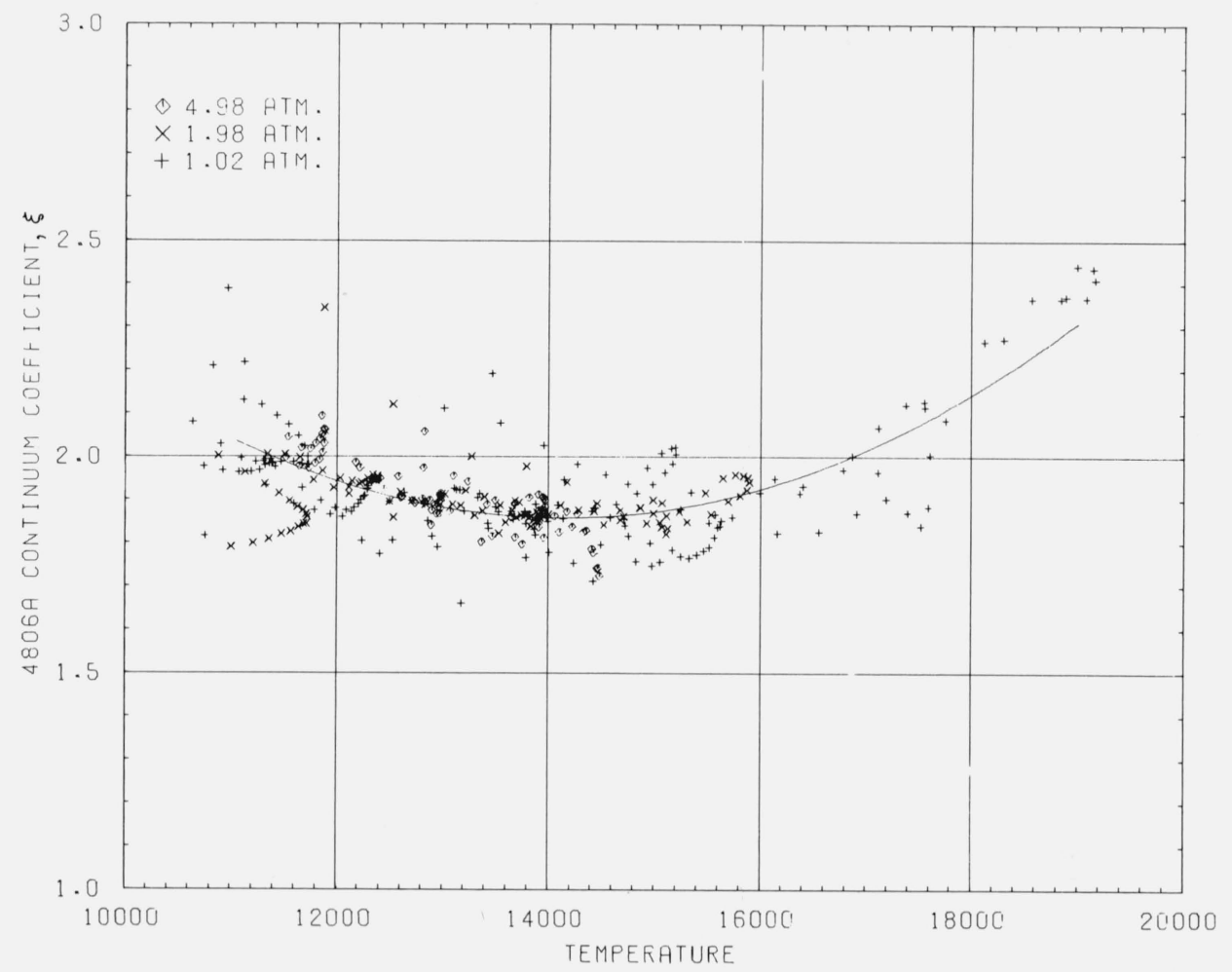

FIGURE 4. Continuum emission correction factors at $4806 \AA$ derived from $1.02,1.98$ and 4.98 atm experiments assuming equilibrium.

The curve is a least squares fitted parabola.

above and values of $\xi$ are given in figure 4. The latter are in fair agreement with Schlüter's theoretical estimate [24] of $\xi=2.2$ at $14000 \mathrm{~K}$. The results of the calculation of $b_{1}$ and $b_{0}{ }^{\mathrm{min}}$ at the axis of the two experiments shown in figure 3 are given in table 1 . The conclusion that, in the $0.5 \mathrm{~atm}$. arc, for example, the neutral excited states are underpopulated by about a factor of 2 and the excited ionic states are further underpopulated by another factor of 2 is in qualitative agreement with the expected non-LTE behavior.

TABLE 1. Departures from equilibrium at axial points of the two experiments plotted in figure 3

\begin{tabular}{c|c|c|c|c}
\hline \hline$P$ & $T_{e}$ & $N_{e}$ & $b_{0} \min$ & $b_{1}$ \\
\hline$(a t m)$ & $K$ & $c m^{-3}$ & & \\
0.5 & 12300 & $3.9 \times 10^{16}$ & 1.9 & 1.9 \\
.2 & 14200 & $2.9 \times 10^{16}$ & 6.6 & 4.2 \\
\hline
\end{tabular}

\section{Summary and Conclusions}

With respect to the question of the existence of LTE, the presentation of arc spectroscopic data in figure 1 can be interpreted to a large extent independently of any assumptions of specific transition probability values. Assuming only that the mechanisms which lead to departures from LTE become less effective as the electron density increases we can conclude that there are substantial departures from LTE in our low pressure argon arcs. Our high pressure arcs are either substantially in LTE or depart from it in a manner which consistently mimics LTE over a surprisingly wide range of conditions. Although one can never expect to obtain complete confirmation of thermodyramic equilibrium we feel that the present study strongly suggests that arcs in argon at electron densities above $5 \times 10^{16} \mathrm{~cm}^{-3}$ are in LTE to within the few percent scatter of our data. This conclusion is based primarily on the apparent disappearance at this electron density of the obvious non-LTE effects of lower electron densities and the reasonable agreement of lifetime measurements with the arc-measured transition probabilities which result from the assumption of LTE. The Ar II transition probability measured here $\left[\mathrm{A}(4806 \mathrm{Ar}\right.$ II $\left.)=7.86 \times 10^{7}\right]$ is in excellent agreement with the lifetime-based value $\left(7.9 \times 10^{7}\right)$ of Wiese's critical compilation [3]. The Ar I transition probability $\left[\mathrm{A}(7147 \mathrm{Ar} \mathrm{I})=5.57 \times 10^{5} \mathrm{~s}^{-1}\right]$ required to fit our intensity measurements under the LTE assumption is smaller than that indicated by any other technique. A comparison of lifetimes for the upper level of this line as measured directly $[25,26]$ and as calculated from theory [27-29] and from this and other emission measurements $[30,31]$ is shown in table 2 . When allowance is made for the possibility of systematic errors in the lifetime measurements and in the arc measurements-both in the absolute 
intensity measurements of the present experiments and in the branching ratio determination - it is doubtful that the differences can be considered significant.

TABLE 2. Ar I $2 p_{2}$ lifetimes

(nanoseconds)

\begin{tabular}{|c|c|}
\hline Klose $[25] \ldots$ & $31 \pm 2$ \\
\hline Verolainen and Osherovich [26].... & $28 \pm 1.4$ \\
\hline Coates and Gaydon $[30] \ldots \ldots \ldots \ldots \ldots$ & $* 26$ \\
\hline Nodwell, Meyer and Jacobson [31].. & 26 \\
\hline Garstang and Van Blerkom [27].... & 29 \\
\hline Johnston $[28] \ldots \ldots \ldots \ldots \ldots . .$. & 30 \\
\hline Meiners $[29] \ldots .$. & 26 \\
\hline Present experiment assuming LTE.. & $* 34$ \\
\hline
\end{tabular}

*Branching ratio taken from arc measurements of ref. [32]

Many argon transition probabilities have been extracted from experiments conducted with a trace of added hydrogen which permits the plasma diagnostics to be based on electron densities derived from $H_{\beta}$ line shape measurements. The resulting transition probabilities, especially for Ar II lines [8,33], are discordant with, for example, the lifetime based values of Wiese's tabulation [3]. If the arcs are in LTE then such experiments indicate that the correct electron density is about 10 percent greater than that obtained from the $H_{\beta}$ line shape measurement and the theoretical profiles of Kepple and Griem [34]. This discrepancy is roughly equal to the estimated uncertainty of the theoretical calculations [34, 35]. On the other hand the same kind of contrived departures from LTE which would create the illusion of LTE in the data of figure 1 but with the wrong transition probabilities would also lead to an apparent constant fractional error in electron density measurements. Again this possibility cannot yet be denied. However, the fact that similar apparent errors in $H_{\beta}$-derived electron density measurements have been observed in many arc gases (hydrogen [36], argon [37, 38], alcohol [39]) tend to support the assumption of arc LTE.

\section{References}

[1] Corliss, C. H., and Shumaker, J. B., Jr., J. Res. Nat. Bur. Stand. (U.S.) 71A, (Phys. and Chem.) No. 6, 575-582, (1967).
[2] Bues, I., Haag, T., and Richter, J., Astron. and Astrophys. 2, 249 (1969).

[3] Wiese, W. L., Smith, M. W., and Miles, B. M., NSRDS-National Bureau of Standards 22, Vol. II (U.S. Government Printing Office, Washington, D.C., 1969).

[4] Olsen, H. N., J. Quant. Spectrose. Radiat. Transfer 3, 305 (1963).

[5] Richter, J., Z. Astrophysik 6 1, 57 (1965).

[6] Bober, L., and Tankin, R. S., J. Quant. Spectrosc. Radiat. Transfer 10,991 (1970).

[7] Shumaker, J. B., Jr., and Popenoe, C. H., J. Opt. Soc. Am. 59, 980 (1969).

[8] Popenoe, C. H., and Shumaker, J. B., Jr., J. Res. Nat. Bur. Stand. (U.S.) 69A, (Phys. and Chem.) No. 6, 495-510 (1965).

[9] Wiese, W. L., Proc. VIII Int. Conf. on Ioniz. Phenom. in Gases, Vienna, 447 (1967).

[10] Wujec, T., Acta Physica Polonica 36,269 (1969).

[11] Griem, H. R., Plasma Spectroscopy, (McGraw-Hill, New York, 1964).

[12] Drawin, H. W., Z. Physik 228, 99 (1969).

[13] Bourasseau, D., Cabannes, F., and Chapelle, J., Astron and Astrophys. 9, 339 (1970).

[14] Desai, S. V., and Corcoran. W. H., J. Quant. Spectrosc. Radiat. Transfer 8, 1721 (1968).

[15] Kolesnikov, V. N., Proc. P. N. Lebedev Phys. Inst. 30, 53 (1966).

[16] Bott, J. F., Physics of Fluids 9, 1540 (1966)

[17] Giannaris, R. J., and Incropera, F. P., J. Quant. Spectrose. Radiat. Transfer 11, 291, 1971.

[18] Uhlenbusch, J., Fischer, E., and Hackmann, J., Z. Physik 239, $120(1970)$

[19] Maecker, H., Z. Natuforsch. 1 la, 457 (1956).

[20] Knopp, C. F., and Cambel, A. B., Rev. Sci. Instr. 37, 332 (1966).

[21] Wolberg, J. R., Prediction Analysis, Van Nostrand, New York, 1967.

[22] Griem, H. R., Phys. Rev. Letters 17, 509 (1966).

[23] Kruger, C. H., Physics of Fluids 13, 1737 (1970).

[24] Schlüter, D.. Z. Astrophysik 61, 67 (1965).

[25] Klose, J. Z., J. Opt. Soc. Am., 57, 1242 (1967).

[26] Verolainen, Ya. F., and Osherovich, A. L., Optics and Spectroscopy (U.S.S.R.) 25, 258 (1968).

[27] Garstang, R. H., and Van Blerkom, J., J. Opt. Soc. Am. 55, 1054 (1965).

[28] Johnston, P. D., Proc. Phys. Soc. (London) 92, 896 (1967).

[29] Meiners, H. M., J. Quant. Spectrosc. Radiat. Transfer 9, 1493 (1969).

[30] Coates, P. B., and Gaydon, A. G., Proc. Roy. Soc. (London) A293, 452 (1966).

[31] Nodwell, R. A., Meyer, J., and Jacobson, T., J. Quant. Spectrose. Radiat. Transfer 10, 335 (1970).

[32] Shumaker, J. B., Jr. and Popenoe, C. H., J. Opt. Soc. Am. 57, 8 (1967).

[33] Berg, H. F., and Ervens, W., Z. Physik 206, 184 (1967).

[34] Kepple, P., and Griem, H. R., Phys. Rev. 173, 317 (1968).

[35] Vidal, C. R., Cooper, J., and Smith, E. W., J. Quant. Spectrosc. Radiat. Transfer 1 1, 263 (1971).

[36] Wiese, W. L., Kelleher, D. C., and Paquette, D. R., submitted to Phys. Rev.

[37] Wende, B., Z. Angew, Phys. 22, 181 (1967).

[38] Morris, J. C., and Krey, R. U., Phys. Rev. Letters 21, 1043 (1968).

[39] Foster, E. W., J. Phys. B3, L145 (1970).

(Paper 76AQ-700) 